Europhysics Letters

PREPRINT

\title{
Charge qubit entanglement in double quantum dots
}

\author{
S. Weiss, M. Thorwart and R. EgGer \\ Institut für Theoretische Physik, Heinrich-Heine-Universität, D-40225 Düsseldorf, Ger- \\ many \\ PACS. 73.21.La - Quantum dots. \\ PACS. 03.65.Ud - Entanglement and quantum nonlocality. \\ PACS. 73.23.-b - Electronic transport in mesoscopic systems.
}

\begin{abstract}
We study entanglement of charge qubits in a vertical tunnel-coupled double quantum dot containing two interacting electrons. Exact diagonalization is used to compute the negativity characterizing entanglement. We find that entanglement can be efficiently generated and controlled by sidegate voltages, and describe how it can be detected. For large enough tunnel coupling, the negativity shows a pronounced maximum at an intermediate interaction strength within the Wigner molecule regime.
\end{abstract}

Semiconductor few-electron quantum dots (QDs) continue to attract a lot of interest, as it has now become possible to experimentally control both the electronic spin and charge in a condensed-phase environment in an unprecedented manner. In particular, double quantum dots (DQDs) can be fabricated in a well-controlled fashion in high-quality semiconductor devices, and are currently under intense study [1-9]. Vertical or lateral tunnel-coupled DQDs are among the most promising candidates for realizing spin or charge qubits in a quantum information processor [10-12]. Their main advantages are scalability, good control of physical properties via tunable external (sidegate-) voltages or magnetic fields, and spatial separation of the individual QDs (allowing to perform one- or two-qubit operations). Recent progress has been very swift, and present-day experiments are performed on DQDs containing just one or two electrons.

We present an exact diagonalization study of ground-state entanglement in a vertical tunnel-coupled DQD containing two interacting electrons. In general, entanglement provides a crucial resource for quantum computing, making certain tasks faster or more secure [13]. While coherent single-electron dynamics has been successfully realized in DQDs, see, e.g., Refs. [8,9], systematic studies of the two-electron dynamics and of entanglement are only now coming into reach [4]. In view of these developments, it seems timely to provide theoretical predictions for two-electron charge entanglement in DQDs, including both the effects of electron-electron interactions and of spin-orbit (SO) couplings. Entanglement of two 'charge qubits', which here arise because an electron may reside in the upper or the lower dot, in such a bipartite mixed state can, for instance, be determined by the Peres-Horodecki measure (the 'negativity' $N$ ) $[14,15]$ in a mathematically sufficient and necessary way. Other entanglement measures exist [16], for instance, the commonly used concurrence $C$ [17], which is mathematically equivalent. It obeys the inequality $C \geq N[18,19]$, although we find only

(c) EDP Sciences 
small deviations between $C$ and $N$ (of order $10^{-3}$ in our case). For sufficiently weak magnetic field, the two-electron system is always in a spin singlet state, and therefore the electrons have opposite spin projections. Since both charge qubits physically occupy the same DQD, this is essential to make entanglement useful: spin projection allows to distinguish different charge qubits, and thereby charge entanglement detection and/or exploitation becomes possible. We outline a concrete proposal below.

Starting from a separable (non-entangled) state in the non-interacting limit, entanglement is enhanced with increasing interaction strength between the two charge qubits. For small tunnel coupling $\Delta$ between the QDs, only very weak interactions are necessary to entangle the qubits, see Eq. (2) below, allowing for efficient entanglement generation. Interestingly, for sufficiently large $\Delta, N$ exhibits a maximum at an intermediate interaction strength, with suppressed entanglement for both weaker and stronger interactions. The 'optimal' interaction strength corresponds to a Brueckner parameter $r_{s} \gtrsim 2$, where Wigner molecule formation is expected $[20,21]$. Our results imply that entanglement of two charge qubits in DQDs can be generated and controlled by gate electrodes, as these affect the lateral size of the QDs and hence the effective interaction strength.

We consider two identical QDs with a two-dimensional (2D) parabolic confinement (in the $x$-y plane) of frequency $\omega_{0}$. In the perpendicular $(z)$ direction, we take a very steep confinement such that only the lowest state $|L\rangle(|U\rangle)$ will be occupied in the lower (upper) QD. For a given electron, this two-level system defines the charge qubit of interest here. We assume a distance $d$ between the QD centers along the $z$ axis, leading to a tunneling amplitude $\Delta$ coupling the QDs. The in-plane coordinates of the two electrons are $\mathbf{r}_{1,2}=\left(x_{1,2}, y_{1,2}\right)$, while the $z$-coordinate is described by the eigenvalue $\tau_{i}= \pm 1$ of a pseudo-spin matrix $\tau^{z}$, where the Pauli matrices $\tau^{x, y, z}$ act in the charge qubit $(|L, U\rangle)$ space. With dielectric constant $\kappa$ of the substrate, effective mass $m^{*}$, and dimensionless Rashba SO coupling $\alpha_{R}$,

$$
\begin{aligned}
H & =\sum_{i=1,2}\left[\frac{\mathbf{p}_{i}^{2}}{2 m^{*}}+\frac{m^{*} \omega_{0}^{2} \mathbf{r}_{i}^{2}}{2}-\frac{\Delta}{2} \tau_{i}^{x}+\omega_{0} l_{0} \alpha_{R}\right. \\
& \left.\times\left(p_{x, i} \sigma_{i}^{y}-p_{y, i} \sigma_{i}^{x}\right)\right]+\frac{e^{2} / \kappa}{\sqrt{\left(\mathbf{r}_{1}-\mathbf{r}_{2}\right)^{2}+d^{2} \delta_{\tau_{1},-\tau_{2}}}}
\end{aligned}
$$

Since the Dresselhaus term or other SO contributions due to the confinement are typically weaker and not tunable by gate voltages, we only keep the Rashba term; Pauli matrices $\sigma^{x, y, z}$ act in spin space. We use $l_{0}=\sqrt{\hbar / m^{*} \omega_{0}}$, the dimensionless interaction parameter $\lambda=e^{2} /\left(\hbar \kappa l_{0} \omega_{0}\right)$ related to $r_{s}[20]$, and put $\Delta=\hbar \omega_{0} \exp \left(-d / l_{0}\right)$, consistent with the $\delta$ function confinement along the $z$-direction.

With integer radial $(n \geq 0)$ and angular momentum $(M)$ quantum numbers, the $\alpha_{R}=$ $\lambda=0$ spin-degenerate single-particle eigenstates to energy $E_{n M}^{ \pm}=(2 n+|M|+1) \hbar \omega_{0} \mp \Delta / 2$ are

$$
\begin{aligned}
\psi_{n M}^{ \pm}(r, \varphi) & =\left(\frac{n !}{2 \pi l_{0}^{2}(n+|M|) !}\right)^{1 / 2} \times(|L\rangle \pm|U\rangle) \\
& \times e^{i M \varphi}\left(r / l_{0}\right)^{|M|} e^{-r^{2} / 2 l_{0}^{2}} \mathcal{L}_{n}^{|M|}\left(r^{2} / l_{0}^{2}\right)
\end{aligned}
$$

with associated Laguerre polynomials $\mathcal{L}_{n}^{m}$. The Hamiltonian (11) is represented in the product basis $\{|U U\rangle,|U L\rangle,|L U\rangle,|L L\rangle\} \otimes\{|\uparrow \uparrow\rangle,|\uparrow \downarrow\rangle,|\downarrow \uparrow\rangle,|\downarrow \downarrow\rangle\} \otimes\left(n_{c}, M_{c}\right) \otimes\left(n_{r}, M_{r}\right)$, where we use center-of-mass $(\mathrm{COM})$ and relative quantum numbers $\left(n_{c, r}, M_{c, r}\right)$, respectively. For $\alpha_{R}=0$, a singlet spin state $S=0$ is realized for arbitrary $\lambda[1]$, and then we restrict the representation 
to this sector. Moreover, the COM part completely decouples in that case. For $\alpha_{R} \neq 0$, however, this decoupling does not hold anymore, no assumptions about the spin sector are allowed, and the full problem has to be diagonalized. (Complete spin entanglement was found for all $\alpha_{R}$ studied below.) In the exact diagonalization scheme, we impose an UV cutoff in $(n, M)$ space and increase it until convergence is achieved. Given the representation of $H$, the antisymmetric two-electron state $\left|\Psi_{0}\right\rangle$ can be computed, and hence the $T=0$ density matrix $\left|\Psi_{0}\right\rangle\left\langle\Psi_{0}\right|$. To study charge qubit entanglement, we form the $4 \times 4$ reduced density matrix $\rho$ by tracing out the spin and $\left(n_{c, r}, M_{c, r}\right)$ degrees of freedom. The Peres-Horodecki measure (i.e. the negativity) then follows as $N=2\left|\zeta_{\min }\right|[14]$, where $\zeta_{\min }$ is the smallest eigenvalue of the partially transposed $\rho^{T_{2}}$ associated with the mixed state described by $\rho$. The partially transposed of the reduced two-particle density matrix $\rho_{\tau_{1} \tau_{2} ; \tau_{1}^{\prime} \tau_{2}^{\prime}}$ (where $\tau=U, L$ ) is defined as $\rho_{\tau_{1} \tau_{2} ; \tau_{1}^{\prime} \tau_{2}^{\prime}}^{T_{2}}=\rho_{\tau_{1}^{\prime} \tau_{2} ; \tau_{1} \tau_{2}^{\prime}}$, i.e. one has to transpose with respect to one of the two electrons only. For mixed bipartite systems, the negativity provides a mathematically rigorous description of entanglement [14-18]. For a separable (non-entangled) state, $N=0$, whereas for maximally entangled states, $N=1$.

Let us then discuss results, starting with $\alpha_{R}=0$. In the absence of interactions, $\lambda=0$, both electrons occupy the same pseudo-spin state $(|L\rangle+|U\rangle) / \sqrt{2}$ with different spin. The two-electron charge state thus factorizes, and $N=0$. In the presence of interactions, however, we find a finite negativity $N$, see Fig. 1, indicating stronger entanglement with increasing $\lambda$ for all $\Delta$ and $0<\lambda \lesssim 1$. This can be rationalized in terms of a simple energy scale argument. For small $\lambda$, only the transverse ground state is occupied $\left(n_{r}=M_{r}=0\right)$. Interactions then tend to localize electrons in the $|L\rangle,|U\rangle$ states, and antisymmetry of the total (spin-singlet) wavefunction implies a symmetric two-electron pseudo-spin state, $\left(\left|L_{1} U_{2}\right\rangle+\left|U_{1} L_{2}\right\rangle\right) / \sqrt{2}$. Tunneling opposes this localization tendency, and a balance is reached for some value $\lambda_{c}$. We take $N\left(\lambda_{c}\right)=1 / 2$ to define $\lambda_{c}$, which sets the crossover scale from weak to strong entanglement. An estimate for $\lambda_{c}$ follows by equating the two relevant energy scales,

$$
\lambda_{c}=\left(\Delta / \hbar \omega_{0}\right)\left|\ln \left(\Delta / \hbar \omega_{0}\right)\right|
$$

Detailed comparison shows that Eq. (2) is in excellent agreement with the values following from the numerical results together with the definition $N\left(\lambda_{c}\right)=1 / 2$. For small $\Delta$, one reaches a maximally entangled Bell state $(N=1)$ already for very weak interactions, see Fig. 1, which demonstrates that charge entanglement can be efficiently generated.

The data shown in Fig. 1(a) reveal an interesting feature for sufficiently large tunnel couplings, $\Delta / \hbar \omega_{0} \gtrsim 0.25$, namely a pronounced entanglement maximum at an intermediate interaction strength $\lambda_{m}>\lambda_{c}$. Note that tunable $\Delta$ reaching a few meV have been reported in recent DQD experiments [3,8]. Since typically $\hbar \omega_{0} \lesssim 10 \mathrm{meV}$, our parameter range for $\Delta$ is accessible. While for $\Delta / \hbar \omega_{0} \lesssim 0.2, N=1$ for $\lambda \gg \lambda_{c}$, in the strong-tunneling case, entanglement decreases again for $\lambda>\lambda_{m}$. The maximum shifts to lower $\lambda_{m}$ when tunneling is increased. To give concrete numbers, $\lambda_{m}=3.53$ for $\Delta / \hbar \omega_{0}=0.37$, while $\lambda_{m}=2.13$ at $\Delta / \hbar \omega_{0}=0.5$, corresponding to $r_{s} \approx 4.8$ and 2.4 , respectively. These interactions indicate that one has reached the incipient Wigner molecule regime [20,21], where interactions become strong enough to induce Wigner crystallization in this finite-size system. The $r_{s}$ values necessary for observing the maximum in $N(\lambda)$ are large but within reach of present experiments [1].

In order to understand this behavior, it is crucial to address the role of higher transverse states in the individual QDs. Those states play no significant role for weak interactions, but can be populated for strong interactions. To elucidate this, consider the following simple DQD 
model with two spin-degenerate levels corresponding to the lower/upper dot,

$$
H_{s}=U_{0} \sum_{j=L, U} n_{\uparrow, j} n_{\downarrow, j}+V_{0} n_{L} n_{U}-\Delta \sum_{\sigma}\left(d_{\sigma, L} d_{\sigma, U}^{\dagger}+\text { h.c. }\right),
$$

where $d_{\sigma j}$ is the annihilation operator for spin $\sigma=\uparrow, \downarrow$ in $\mathrm{QD} j=L / U, n_{\sigma j}=d_{\sigma j}^{\dagger} d_{\sigma j}$, and $n_{j}=n_{\uparrow, j}+n_{\downarrow, j}$ with $n_{L}+n_{U}=2$. The interaction between electrons on the same dot (on different dots) is $U_{0}\left(V_{0}\right)$. The negativity can then be calculated in the same way as before and is shown as a function of $U_{0}$ in Fig. 2, with the order-of-magnitude estimate $V_{0}=U_{0} /\left(d / l_{0}\right)$. These results indicate that for any tunneling amplitude $\Delta$, entanglement becomes stronger with increasing interactions. The same conclusion is reached by using more sophisticated estimates for $U_{0}, V_{0}$, starting from Eq. (1) and projecting the full Hamiltonian to the transverse ground state. We conclude that $H_{s}$ is not able to reproduce the suppression of entanglement at large $\lambda$ seen in Fig. 1(a), and therefore higher transverse states of the QDs are crucial in understanding this effect. In fact, keeping only oscillator states with $n_{r} \leq n$ in the diagonalization of Eq. (1), Fig. 2(b) shows that with increasing $\lambda$ higher states become more and more relevant. For $\Delta / \hbar \omega_{0}=0.37$, see Fig. 2(b), we recover the entanglement maximum once $n \geq 3$. The relevance of these higher-lying transverse states for the suppression of entanglement also follows from an energetic argument involving the competition of tunneling and Coulomb repulsion. For this, consider the energy difference

$$
\varepsilon_{\min }(\lambda, \Delta)=E_{\text {same }}-E_{\text {diff }},
$$

where the two electrons either reside on the same dot $\left(E_{\text {same }}\right)$ or on different dots $\left(E_{\text {diff }}\right)$. For $\lambda=0$, we have $\varepsilon_{\min }=0$, corresponding to degenerate pseudo-spin states and therefore to $N=0$. In general, we can then expect that a correlation between $N$ and $\varepsilon_{\min }$ exists, with large (small) $\varepsilon_{\min }$ corresponding to large (small) $N$. This correlation is natural [11], since the entangled state corresponds to the electrons occupying different dots, leading to the energy $E_{\text {diff }}$, while entanglement is destroyed once both electrons reside on the same dot. Diagonalizing $H$ separately for these two cases, the numerical result for $\varepsilon_{\min }$ is shown for various $\Delta$ as a function of $\lambda$ in Fig. 1(b). The energy difference $\varepsilon_{\min }$ grows with increasing $\lambda$ for $\Delta / \hbar \omega_{0} \lesssim 0.2$, but has a maximum for $\Delta / \hbar \omega_{0} \gtrsim 0.25$. Remarkably, the behavior of $\varepsilon_{\min }$ perfectly reflects what we obtain for the negativity. The decrease in $\varepsilon_{\min }$ with large $\lambda$ does not occur for $H_{s}$ (data not shown), which again points to the importance of higherlying transverse states. Intuitively, the interaction energy is minimized by pushing the two electrons to opposite sides in the $x-y$ plane, which corresponds to occupation of these states. By that mechanism, the Coulomb repulsion between electrons located on different dots is efficiently reduced, and thereby entanglement can be suppressed. We mention that for very large interactions, a simple classical calculation (i.e., neglecting the kinetic energy) shows that $\varepsilon_{\min }$ reaches a constant value, $\varepsilon_{\min } \propto d^{2}$. This in turn implies by the above argumentation that the negativity does not vanish as $\lambda \rightarrow \infty$, but instead stays at a finite value.

Let us then briefly address the effects of finite SO couplings $\alpha_{R}$ on charge entanglement, see Fig. 3, In general, SO couplings tend to weakly decrease $N$. For InAs dots, where SO couplings are expected to be quite strong, values in the regime $\alpha_{R} \lesssim 1$ were measured [22]. For such couplings, we find that $N$ decreases by at most $20 \%$. The decrease is most pronounced for strong tunneling between the dots and/or strong electron-electron interaction. In the presence of various types of SO couplings, already for just one electron in the DQD one may expect 'hyperentanglement' [23] of spin and charge. However, keeping only the Rashba coupling in Eq. (1) does not lead to a non-zero hyperentanglement negativity, even when allowing for different SO couplings in the two dots. 
When tuning entanglement by adiabatic manipulations such as slow changes of gate voltages, some relaxation mechanism is implicitly needed for equilibration. However, we require relaxation to be weak enough, for otherwise quantum coherence is affected or even destroyed. Typical charge relaxation times in high-quality DQDs presently approach $1 \mu \mathrm{s}$ [9], and thus the coherence requirements should pose no major obstacle. Let us then discuss how the charge qubit entanglement produced in the DQD can be detected and/or exploited by application of the scheme suggested in Ref. [24], see Fig. 4. Two electronic beam splitters [25] are attached together with two side gates in one input arm of each. The latter allow to induce controlled phase shifts $\phi_{A}$ and $\phi_{B}$ on the orbital states [26]. The accessible observables are average currents $I_{\nu}^{\alpha}$ and zero-frequency current correlators $S_{\nu, \mu}^{\alpha, \beta}$ with $\nu, \mu=A, B$ and $\alpha, \beta= \pm$, see Fig. 4 Suppose now that the contacts to the beam splitters can be individually addressed by adiabatic gate voltage pulses switching them from 'closed' to 'open'. Two electrons are then emitted from the double dot and enter the detection region. The two-particle reduced density matrix (and hence $N$ ) then follows from 16 current correlation and 8 average current measurements [24].

To conclude, we have studied charge qubit entanglement in double quantum dots. Entanglement can be created and altered by adiabatic changes of electrostatic potentials. While we have specifically discussed vertical double dots, our general conclusions also apply to lateral double dots or carbon-nanotube based dots. The case of more than two interacting electrons remains as an interesting open challenge, where the mathematical foundations for entanglement measures are less clear.

We thank D. Bruß, T. Heinzel, and H. Kampermann for discussions. This work was supported by the ESF network INSTANS.

\section{REFERENCES}

[1] van der Wiel W. G., De Franceschi S., Elzerman J. M., Fujisawa T., Tarucha S., and Kouwenhoven L., Rev. Mod. Phys., 75 (2003) 1.

[2] Elzerman J. et al., Phys. Rev. B, 67 (2003) 161308(R).

[3] Hatano T., Stopa M., and Tarucha S., Science, 309 (2005) 268.

[4] Petta J. R. et al., Science, 309 (2005) 2180.

[5] Johnson A. C. et al., Nature, 435 (2005) 925.

[6] Koppens F. H. L. et al., Science, 309 (2005) 1346.

[7] Krenner H. J.et al., Phys. Rev. Lett., 94 (2005) 057402.

[8] Hüttel A. K., Ludwig S., Lorenz H., Eberl K., and Kotthaus J., Phys. Rev. B, 72 (2005) $081310(\mathrm{R})$.

[9] Gorman J., Hasko D. G., and Williams D. A., Phys. Rev. Lett., 95 (2005) 090502.

[10] Loss D. and DiVincenzo D. P., Phys. Rev. A, 57 (1998) 120.

[11] Burkard G., Seelig G., and Loss D., Phys. Rev. B, 62 (2581) 2000; Golovach V. N. and Loss D., Phys. Rev. B, 69 (2004) 245327.

[12] Schliemann J., Loss D., and MacDonald A. H., Phys. Rev. B, 63 (2001) 085311.

[13] Nielsen M. A. and Chuang I. L., Quantum Computation and Quantum Information (Cambridge University Press) 2000.

[14] Peres A,. Phys. Rev. Lett., 77 (1996) 1413; Horodecki M., Horodecki P., and Horodecki R., Phys. Lett. A, 223 (1996) 1.

[15] Thorwart M. and Hänggi P., Phys. Rev. A, 65 (2002) 012309.

[16] Bruss D., J. Math. Phys., 43 (2002) 4237.

[17] Wootters W. K., Phys. Rev. Lett, 80 (1998) 2245.

[18] Verstraete F., Audenaert K., Dehaene J., and De Moor B., J. Phys. A, 34 (2001) 10327.

[19] The measure introduced in Ref. [12] here equals $C$. 


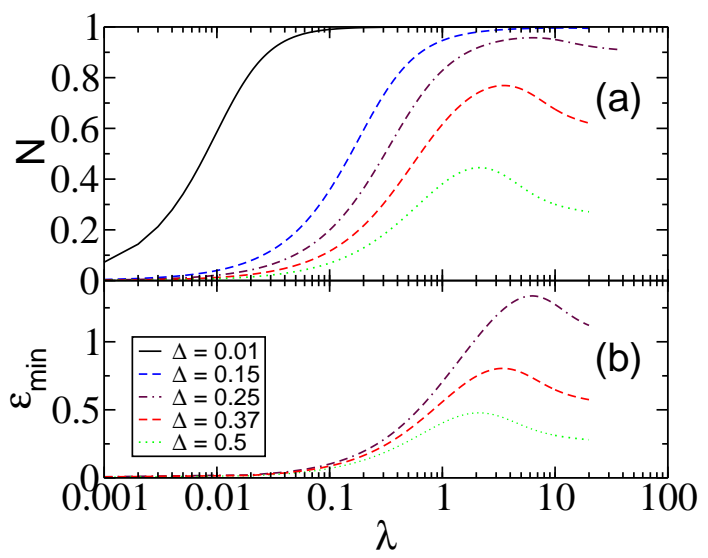

Fig. 1 - (Color online) (a) Negativity $N$ versus interaction strength $\lambda$. Results are shown for several $\Delta$ (in units of $\hbar \omega_{0}$ ), with $\alpha_{R}=0$. (b) Corresponding energy scale $\varepsilon_{\min }$ (in units of $\hbar \omega_{0}$ ), see Eq. (4), as a function of $\lambda$. (Data for $\Delta / \hbar \omega_{0} \leq 0.15$ are not shown, since $\varepsilon_{\text {min }}$ has no maximum.) Note the semi-logarithmic scale.

[20] Reimann S. M. and Manninen M., Rev. Mod. Phys., 74 (2002) 1283.

[21] Weiss S. and Egger R., Phys. Rev. B, 72 (2005) 245301.

[22] Nitta J. et al., Phys. Rev. Lett., 78 (1997) 1335; Koga T. et al., Phys. Rev. Lett., 89 (2002) 046801.

[23] Walborn S. P., Padua S., and Monken C.H., Phys. Rev. A, 68 (2003) 042313.

[24] Samuelsson P. and Büttiker M., Phys. Rev. B, 73 (2006) 041305(R).

[25] Henny M. et al., Science, 284 (1999) 296; Oliver W. D., Kim J., Liu R., and Yamamoto Y., Science, 284 (1999) 299.

[26] Ji Y. et al., Nature, 422 (2003) 415.

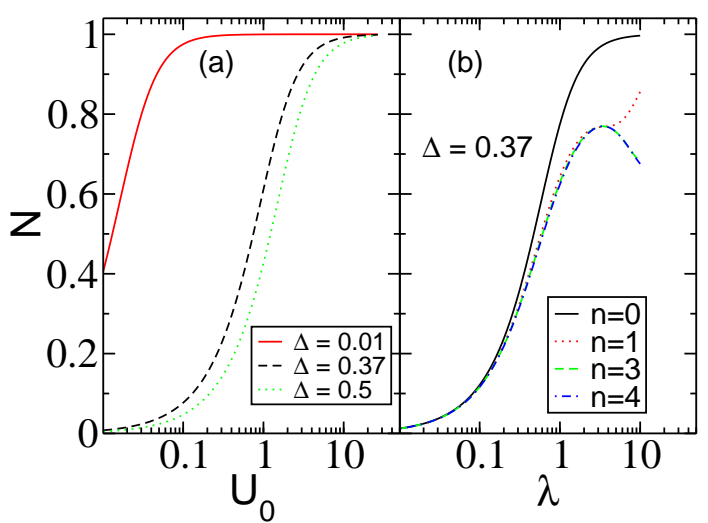

Fig. 2 - (Color online) Negativity versus interaction strength for (a) the simple model $H_{s}$ in Eq. (3), with several different $\Delta$ (in units of $\hbar \omega_{0}$ ), and (b) for the full $H$ at $\Delta / \hbar \omega_{0}=0.37$, with the number of oscillator states $n_{r}$ truncated to $n_{r} \leq n$ (for several $n$ ). 
S. Weiss, M. Thorwart and R. Egger: Charge Qubit entanglement in double Quantum Dots7

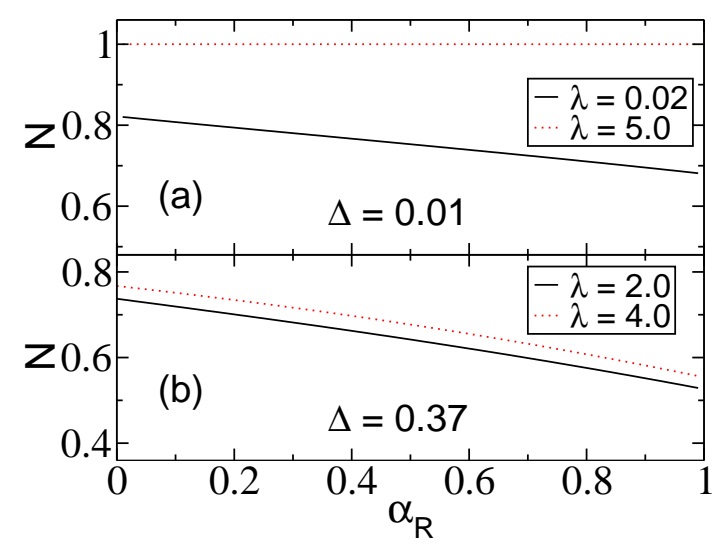

Fig. 3 - (Color online) Negativity as function of the SO coupling $\alpha_{R}$ for several $\lambda$, for (a) $\Delta / \hbar \omega_{0}=0.01$ and (b) $\Delta / \hbar \omega_{0}=0.37$.

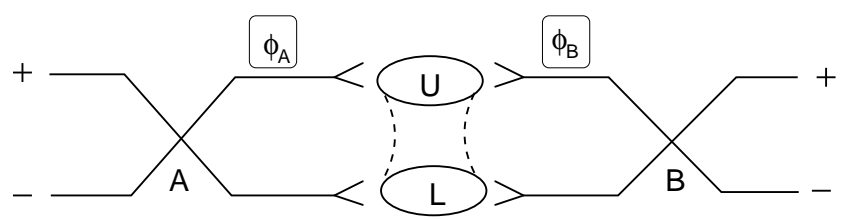

Fig. 4 - Schematic setup to detect charge qubit entanglement adopted from Ref. [24]. The upper and lower dot in the DQD are indicated by $U$ and $L$, respectively. The DQD is connected by switchable barriers to two beam splitters $A$ and $B$, and two side gates induce controlled phase shifts $\phi_{A / B}$. 